\title{
QUIEBRE AL CENTRALISMO REGIONAL: PROVINCIA DE ILO AVANZA CON MAYOR INVERSIÓN PRIVADA Y PBI, 2001-2019
}

\author{
BREAK TO REGIONAL CENTRALISM: ILO PROVINCE ADVANCES WITH GREATER \\ PRIVATE INVESTMENT AND GDP, 2001-2019
}

\section{RESUMEN}

Objetivo: Demostrar que la provincia de llo, no siendo capital, centraliza la inversión privada, el empleo y el valor agregado; mayor que en los otros 19 distritos de la región Moquegua en el periodo 2001-2019. Método: Es un estudio básico, explicativo, no experimental, se analiza los censos económicos del 2001 y 2008, datos del Banco Central de Reserva del Perú, Instituto Nacional de Estadística e Informática y aplicado los estadísticos R, R2, y ANOVA, en los Software SPSS 25 y Eviews 10. Resultados: Ilo en 1940 tenía 1656 habitantes y 66876 en el año 2018, en 78 años se ha multiplicado por 40 . El número de bancos es mayor que en 18 distritos. En el censo económico 2008, la provincia de llo concentraba el mayor número de empresas, empleos, activos y patrimonio; después del 2008, esta concentración se ha incrementado en términos relativos, aunque no en absolutos. En el año 2019, llo concentra el 68,8\% de los créditos de la región. Conclusiones: Existen diferencias en los niveles de inversión expresada en colocaciones, en el valor bruto de la producción entre los distritos de llo y Moquegua. Las tasas de crecimiento de los créditos en llo, son mayores que en los demás distritos.

Palabras clave: Créditos; centralismo distrital; concentración económica; desarrollo; inversión privada.
Javier Pedro Flores Arocutipa Universidad Nacional de San Agustín Arequipa, Perú ORCID: https://orcid.org/0000-0003-0784-4153 Correo electrónico: doctorjavierflores@hotmail.com

Julio César Luján Minaya Universidad Nacional de Cañete Lima, Perú

ORCID: https://orcid.org/0000-0003-3752-824X Correo electrónico: aguila_jclm2013@hotmail.com

JoRGE JinCHUÑA HUALLPA Universidad Nacional de Moquegua Moquegua, Perú

ORCID: https://orcid.org/0000-0002-9073-3798

Correo electrónico: jjinchunah@unam.edu.pe

[Recibido: 24/07/2020 Aceptado: 11/12/2020 Publicado: 28/12/2020]

\begin{abstract}
Objective: To demonstrate that the llo province, not being capital, centralizes private investment, employment and added value; more than in other 19 districts of Moquegua region in the period 2001 - 2019. Method: It is a basic, explanatory, non-experimental study, the economic censuses of 2001 and 2008 are analyzed data from the Central Reserve Bank of Peru, the National Institute of Statistics and Informatics and the statisticians R, R2, and ANOVA were applied, in the SPSS 25 and Eviews 10 Software. Results: Ilo in 1940 had 1656 inhabitants and 66,876 in 2018, in 78 years it has multiplied by 40 . The number of banks is higher than in 18 districts. At the 2008 economic census, the province of llo concentrated the largest number of companies, jobs, assets and assets; after 2008, this concentration has increased in relative terms, not absolute though. In 2019 , llo concentrates the $68.8 \%$ of the region's credits. Conclusions: There are differences in the investment levels expressed in placements, in the gross value of production between the districts of Ilo and Moquegua. Credit growth rates in llo are higher than in other districts.
\end{abstract}

Keywords: Credits; district centralism; economic concentration; development; private investment. 


\section{INTRODUCCIÓN}

El centralismo y la concentración económica son temas de larga data. Las leyes de descentralización y de gobiernos subnacionales señalan que se debe promover el desarrollo armónico de las regiones, provincias y distritos (Jurado Nacional de Elecciones, 2002). La razón es que ello garantiza mayor equidad, igualdad y mejor calidad de vida de las personas (Miranda, 2017). Esta situación no se nota entre las ciudades de Ilo, Moquegua y los demás distritos. Se está creando un desarrollo diferenciado entre distritos, fuera de Ilo y Moquegua y entre estas. Así en 2019, en el distrito de Ilo se concentra el $68,8 \%$ de los créditos otorgados en la región Moquegua. El distrito Moquegua concentra el 32\% de los créditos.

La ciencia económica señala que el crecimiento armónico de las inversiones permite proyectar la calidad de vida, incrementando los ingresos per cápita, el PBI per cápita. Así, en el estudio Impacto de la expansión de las colocaciones de las instituciones micro financieras en el crecimiento Regional del Perú en el período 2001 - 2011 se demostró que los créditos sí influyen, que sí explica el crecimiento del PBI. Asimismo, demuestra que a un incremento del $10 \%$ de las colocaciones, el crecimiento del PBI sería de $0,69 \%$. Si el crecimiento de los créditos fuera del $20 \%$ es posible que el impacto en la economía regional sería de $1,4 \%$ (Galicio, 2013).

En ese mismo sentido, Tapia (2018) aplicando el modelo de crecimiento de Ramsey, demuestra que sí hay correlación entre las variables de créditos y crecimiento del PBI. De modo semejante, Aguilar (2019) demostró que hay impacto de los créditos en el crecimiento económico y el desarrollo financiero en el período 2001-2008. En esa ruta, se entiende que los créditos se relacionan con el crecimiento económico y en muchos casos la primera variable explica la segunda.

Los créditos en el Perú, desde hace buenos años, se concentran en Lima, esto no es desconocido. A noviembre de 2019, Lima concentraba 240,3 mil millones de soles, y eso representa el 73,07\%. Le sigue Arequipa con el 3,6\%, La libertad con el 2,84\% (9351 millones). Moquegua representa el 0,46\% (1521,3 millones). Asimismo, la región Tacna posee el 0,79\% (2608 millones). Se observa que Lima ha crecido porcentualmente y en términos absolutos y que hoy tiene una mayor concentración de los créditos (Banco Central de Reserva del Perú [BCRP], 2019).

Moquegua no supera el 0,46\%, aunque ha mejorado desde que era el 0,33\%, hace quince años atrás. Las regiones de Huancavelica, Amazonas, Pasco y Madre de Dios, representaron no más del 0,19\%. Mientras que Puno Cajamarca, Áncash, Ica, Cusco, Junín y Lambayeque tienen entre el 1 y $2 \%$ de los créditos totales. Hay un total de créditos en el Perú de 315 mil millones de soles al cierre del año 2019.

La economía peruana no es una economía dolarizada, dado que solo el $27 \%$ de los créditos son en moneda extranjera. Aclarando que las economías que tienen créditos en moneda extranjera, y más representativas son: Lima con $33 \%$, Callao con $30 \%$, Ica con $23 \%$, Arequipa con $11 \%$ y La libertad con $12,1 \%$. Se nota que los créditos y su concentración son una expresión de la distribución de la producción y riqueza en el Perú.

\section{MATERIALES Y MÉTODOS}

El tipo de investigación es básica, no se manipulan escenarios. La realidad es un hecho. Los datos de créditos y depósitos se acopiaron del Banco Central de Reserva del Perú (BCRP) en relación a cada uno de los distritos donde se hayan realizado los depósitos y colocaciones en el período 2001-2019. Es una serie periódica, longitudinal, por ello se analiza con los estadísticos $\mathrm{R}, \mathrm{y} \mathrm{R}^{2}$ y ANOVA. Tiene el nivel de investigación relacional, explicativo y comparativo. Es no experimental, de fuente primaria y secundaria, se ha entrevistado a las principales autoridades en opinión de expertos.

La población es la base de datos histórica de depósitos y créditos hasta el año 2019. La muestra de trabajo va de los años 2001 al año 2019, son 19 años de análisis. Se trabajó la base de datos de la región Moquegua y sus distritos. El instrumento utilizado es la ficha de investigación, en la que se ha vaciado los datos requeridos y necesarios para el análisis de los créditos en los distritos de Ilo y Moquegua. En ella también se ha determinado, en base a la tasa de crecimiento, cómo el distrito de Ilo ha logrado concentrar y crecer en lo que respecta a colocaciones y depósitos. Y en base a los censos nacionales del año 1993 y el censo económico del 2008 se observa cómo ha consolidado su crecimiento y cómo se ha hecho consistente la dinámica económica en dicha provincia.

En el programa Excel se ha preparado y presentado la base histórica de la data, para luego ser procesada en el programa SPSS 25 y en el programa EVIEWS 10. Así se pudo demostrar si realmente hay diferencias entre los datos obtenidos de concentración y crecimiento económico entre Ilo, Moquegua y distritos. Se revela si hay diferencias entre los datos que se van a comparar, entonces se puede afirmar que una tiene mejor crecimiento y además concentra los créditos de la región Moquegua.

\section{RESULTADOS}

El centralismo limeño, el centralismo arequipeño y el centralismo regional en Moquegua: los créditos. La información 
mostrada indica que en el 2019 ha crecido el volumen de créditos en el país. Así, Lima en el 2019, contaba con 240 mil 299 millones de soles en créditos. Constituyendo el 92,3\% del total de créditos en la tabla 1.

Moquegua también ha incrementado su participación en el mercado de créditos, al 2019 tenía 1521 millones. Tacna crece en colocaciones de 1453 millones de soles en 2004 a 2608 millones en el 2019. Puno crece de 2255 millones a 3806 millones en el 2019. Arequipa al cierre del año 2019 tiene créditos por 11847 millones de soles.

Se observa que hay niveles de concentración de riqueza. Se tiene que Lima es 158 veces la dinámica económica de Moquegua. Arequipa es 8 veces, Puno tres veces, Tacna dos veces el movimiento de bienes y servicios.

Arequipa lidera los créditos en el sur peruano. Lima es al Perú como Arequipa es al sur peruano. Al igual que en el Perú hay concentración de capitales, del mismo modo en el sur peruano hay concentración de riqueza y Arequipa se ha convertido en un eje de la dinámica del sur. Al cierre del año 2019 tenemos lo siguiente: Arequipa tiene créditos colocados en un $41,1 \%$ del total, Cusco el $19,87 \%$, Puno el $12,23 \%$, Tacna el $9,16 \%$ y Moquegua el 5,33\%. Considerando que el total de créditos distribuidos asciende a 27 mil 500 millones de soles.
La pregunta es: ¿Qué ha hecho consistente que Ilo poco a poco vaya posicionándose de la riqueza regional en Moquegua? Una forma de medir ello, son los depósitos y las colocaciones, el número de entidades financieras y oficinas que se apertura en los distritos. Hay una relación alta y significativa entre PBI regional y créditos por región. Así los datos del PBI del 2018 y créditos de noviembre por regiones del 2019 generó un coeficiente de correlación de $99,2 \%$ y un coeficiente de determinación de 98,44\%. Quiere decir que a mayores créditos, léase inversión privada, hay mayores niveles de PBI por regiones.

En la provincia de Ilo, la población en el año 1993 era de 53 574 habitantes y en el 2018 de 66874 habitantes. Según los censos económicos de 1993 y el 2008 los establecimientos (empresas) en el año 1993 eran 2661, en la región y de ellos, 1163 estaban en la provincia de Ilo, (representaba el 43,7\%). El número de establecimientos en el año 2008 en Ilo era de 3651 y representaba el 54\%. En la práctica la mayoría de establecimientos ya estaban en Ilo. Se nota que la densidad de establecimientos por habitantes se había incrementado en 55 por cada 100 habitantes, mientras que Mariscal Nieto se quedaba en 37 establecimientos por cada 100 habitantes. Aquí resalta la relación de mayor negocio y menor población.

Tabla 1

La concentración de capitales (en miles de soles) en el sur peruano

\begin{tabular}{lrrrcc}
\hline Regiones & \multicolumn{1}{c}{ 2004 Dic } & 2012Dic & 2019Nov & \% & Veces \\
\hline Lima & 29691843 & 115674901 & 240299330 & 92,39 & 158 \\
Arequipa & 1433685 & 6493703 & 11847392 & 4,56 & 8 \\
Puno & 209645 & 2255568 & 3806305 & 1,46 & 3 \\
Tacna & 240788 & 1453815 & 2608298 & 1,00 & 2 \\
Moquegua & 130075 & 553023 & 1521369 & 0,58 & 1 \\
TOTAL & 31706036 & 126431010 & 260082694 & 100 & \\
\hline
\end{tabular}

Fuente: Elaboración propia con información de BCRP. Miles de soles

Tabla 2

Niveles de correlación de variables

\begin{tabular}{lccc}
\hline & MOQUEGUA & ILO & Región \\
\hline CARUMAS & 0,670 & 0,593 & 0,627 \\
TORATA & 0,498 & 0,328 & 0,392 \\
PUQUINA & 0,756 & 0,787 & 0,783 \\
PACOCHA & $-0,195$ & $-0,164$ & $-0,177$ \\
OMATE & 0,918 & 0,873 & 0,897 \\
MOQUEGUA & 1,000 & 0,959 & 0,982 \\
ILO & 0,959 & 1,000 & 0,995 \\
Región & 0,982 & 0,995 & 1,000 \\
\hline
\end{tabular}

Fuente: Elaboración propia utilizando Eviews 10. SBS 
El IV censo económico del año 1995 revela que en la región se aperturaron operaciones por 1178 establecimientos, de ellos en Ilo habían 596. Disminuye en los años de 1995 a 1999 y de un total de 852 empresas que iniciaban operaciones, en Ilo lo hacían el 50,5\% del total. En el periodo del 2000 al año 2004, los establecimientos eran 1438 y a Ilo le corresponde el 55,35\%. En el 2005 (56,1\%); 2006 (56,87\%); 2007 (58,39\%); 2008 (53,51\%) este es el crecimiento, cada vez de mayor apertura a los negocios, que permite observar que en el periodo de 1995 al año 2008, del acumulado de 6757 empresas nuevas, 3651 empresas corresponden a la provincia de Ilo y 2793 a la provincia de mariscal Nieto. Ilo ya tenía acumulado el $54 \%$ y Mariscal Nieto el 41,3\%. Las diferencias se dejaban notar.

En el censo del año 2008, en el sector extractivo, de un total de 336 establecimientos 331 (98,5\%) estaban en Ilo. En el sector trasformación, de 425 empresas, 275 eran de Ilo $(47,8 \%)$; y en servicios de la misma manera, de un total de 425 empresas 203 correspondían a Ilo (47,8\%). Podemos afirmar que las microempresas vinculadas a la pesca son mayores en Ilo.

La formalización es vital para el crecimiento económico y sobre todo porque marcha hacia la bancarización.

En producción censal, si bien las empresas declarantes en el año 2007 fueron 5258 empresas en ese entonces 2767 eran de Ilo (52,62\%); de la provincia de Mariscal Nieto fueron 2256 empresas informantes (42,9\%).

Si la producción censal de Moquegua era de 10111 millones 789 mil soles, la provincia de Ilo producía $7 \mathrm{mil} 96 \mathrm{mi}$ llones 980 mil soles (70,2\%). No había lugar a duda que en el 2007 se percibe silenciosamente que la provincia de Ilo se había posicionado de la región en producción censal.

Se ha señalado que el consumo es vital para el crecimiento económico como primera variable, este es favorable a Ilo, de 3 mil 67 millones 849 mil soles de Consumo Intermedio Censal, el 69,6\% estaba en Ilo; en Mariscal Nieto, $30,3 \%$ y en General Sánchez Cerro el 0,1\%.

El valor agregado (VAC), que son los pagos que se hacen a los factores de la producción, la cadena de valor. Entonces de un VAC de 7 mil 43 millones 940 soles de la región, el 70,4\% le corresponde a Ilo, el 29,5\% a Mariscal Nieto y el 0,1\% a General Sánchez Cerro (5258 empresas informantes).

Debemos centrar nuestra mirada en los resultados antes de la participación del impuesto censal porque ella resulta ser en Ilo de cuatro mil 45 millones 337 mil soles, que representa el $71,2 \%$ del total regional. Y en utilidades del ejercicio de las empresas en la región, Ilo concentraba el 71\%.
Y la pregunta es: ¿Dónde están los activos? La respuesta es, en el año 2007, de activos valorizados por 21 mil 378 millones 663 mil soles, el 73,7\% se encontraba en Ilo y el 26,3\% en la provincia de Mariscal Nieto.

La depreciación es el factor que mide cuánto es el desgaste de maquinaria, equipo, edificio y eso dependerá de su tamaño y utilización. La depreciación del periodo, fue 254 millones 972 mil soles y el 74\% se generó en Ilo; y en el caso la depreciación adicional, Ilo (2007) representó 25 millones 843 mil soles que es el 83,9\% de la región. De tal manera que de los saldos finales que fueron de $6 \mathrm{mil} 6 \mathrm{mi}$ llones de soles el 73,8\% correspondía a la provincia de Ilo.

¿Y el empleo? De las 5258 empresas informantes, se obtuvo que el empleo generado en ese año fue de 20749 puestos laborales. De ellos el 64,5\% se ubicaban en Ilo (13 378) y el 33,6\% en la provincia de Mariscal Nieto (6975) (Instituto Nacional de Estadistica e Informática [INEI], 2020).

El año 2008 el capital humano, en los 20 distritos de Moquegua, el distrito de Pacocha ocupaba el primer lugar en el ranking (población de más de 15 años con educación superior), el porcentaje era el 51\%, seguido de Omate con el $40 \%$, el distrito de Algarrobal ocupaba el 5to lugar con $34 \%$, el distrito de Ilo estaba en el puesto siete con el 33\% de capital humano, y el distrito de Coalaque ocupaba el último lugar con el $9 \%$.

Respecto a las Tecnologías de la Información y la Comunicación (TIC) y computadoras, el indicador es el número de computadoras por viviendas en porcentaje. En ese extremo, el distrito de Pacocha tenía el 45\% de viviendas con al menos una computadora, le seguía el distrito de Ilo con el 16\%, Moquegua con 15\%, Samegua con el 15\%, y así se llega al distrito número 13, Carumas con al menos $1 \%$ de hogares que tenía una computadora. Y se deja de contar.

En cuanto al número de celulares por vivienda, de la misma manera el 2008, Pacocha, tenía el 63\% de viviendas con un celular, Ilo el 62\%, el distrito de Moquegua el 52\%, el distrito de Samegua el 48\% el distrito de Torata el 35\% y así el distrito de Coalaque, que ocupaba el puesto número 8 tenía el 1\% de vivienda con al menos un celular.

Otra de las preocupaciones es el servicio de Internet. En el censo del 2008, en Pacocha el 26\% los hogares tenían el servicio de internet. En segundo lugar, estaba Ilo con el 7\%. El Distrito de Moquegua tenía el 4\% de hogares con internet, así como el distrito de Torata (4\%), el último distrito que registra el servicio es el Algarrobal con el 1\%.

El índice de Desarrollo Humano (IDH) es un indicador de calidad de vida, de tal manera que en el año 2003 el IDH por provincias era: Ilo con un IDH de 0,45; Mariscal Nieto 
0,424 y General Sánchez Cerro 0,379 y en el ranking nacional Ilo se ubicaba en el puesto 10 mientras que la provincia de Mariscal Nieto en el puesto 15 y Gral. Sánchez Cerro en el puesto 30. En el 2012 tal situación varía de tal manera que la provincia de Ilo ocupaba el primer lugar a nivel nacional con 0,668 de IDH, mariscal Nieto estaba en el segundo lugar y Gral. Sánchez Cerro en el puesto 44. Por distritos, Pacocha ocupa el puesto dos a nivel nacional con 0,7920 de IDH, le sigue el Algarrobal con 0,7075 en el puesto 20. Ilo por supuesto se ubicó en esa época en el puesto 37 con un IDH de 0,6598. El distrito de Torata se ubica en el puesto $27 \mathrm{y}$ el distrito de Moquegua en el puesto 36 . Omate en el puesto 186 con un IDH de 0,5273 .

El consumo se basa en la población. Ilo en 1940 tenía una población de 1656 habitantes. Al censo del año 1961 era de 10 215. En 1972 la población fue de 25187 personas. En 1981 la población de Ilo era de 38627 habitantes, en el año 1993 ya era 51481 habitantes con un crecimiento anual de 1071 personas, en el año 2017 se contaban 63780 personas (INEI, 2020).

En lo que se refiere a la inversión privada medida por créditos en los distritos de Moquegua en 2019, encontramos que:

- $\quad$ La Correlación de Pearson (CP): entre créditos Ilo con los créditos totales de la región es de 99,5\%, lo mismo, la CP de créditos de Moquegua con el total es de 98,2\%, los créditos del distrito de Carumas se relaciona con el total Moquegua en 62,7\%, los créditos del distrito de Omate con el total son de $39,2 \%$ y en el caso del distrito de Pacocha la CP es de $-0,17$ lo que quiere decir que la relación es inversa entre estas dos variables.

- De la misma manera, la CP de las colocaciones en los distritos de Moquegua e Ilo es de 95,9\%, que da a entender que los créditos corren en la misma dirección y son casi paralelas. (Ver tabla 3)

Tabla 3

Variable dependiente: Créditos en la región

Muestra: 2001-2019

\begin{tabular}{lccc}
\hline Variable & Coefficient & t-Statistic & Prob. \\
\hline C & $-51,25$ & $-0,07$ & 0,94 \\
Créditos llo & 0,99 & 206,68 & 0,00 \\
Créditos Moquegua & 1,04 & 108,14 & 0,00 \\
R-squared & 1,00 & & \\
F-statistic & 611145 & & \\
Prob(F-statistic) & 0 & & \\
$\quad$ Durbin-Watson & 1,736 & & \\
\hline
\end{tabular}

Fuente: Elaboración propia Eviews 10. SBS
- De otro lado, en la tabla 3 podemos observar que el crecimiento de los créditos en los distritos de Ilo y en Moquegua, de esa manera se ha encontrado la regresión: Total de Créditos = $-51,2548577302+0,992559908965^{\star}$ Créditos ILO $+1,03882027163^{\star}$ Créditos MOQUEGUA, su nivel de explicación está determinado por el $\mathrm{R}^{2}$ $=0,9999$ que es alto y significativo. Que los coeficientes tienen relevancia dado que las betas de las variables tienen una probabilístico menor de 0,05 ; además que el $\mathrm{F}$ probabilístico nos indica que es una buena ecuación que interpreta de manera coherente el crecimiento del nivel de créditos en la región.

- Otro de los indicadores que se debe tomar en cuenta es el nivel de impacto de las colocaciones en los distritos de la región. Para ello utilizaremos el concepto de elasticidad con logaritmos. El impacto del crecimiento del $1 \%$ de los créditos en el distrito de Ilo permite un crecimiento del 0,77\% de los créditos en la región. Si el incremento fuera del 10\% en el distrito de Ilo, el crecimiento de créditos en la región sería del 7,7\%.

- Si el incremento de los créditos en el distrito de Moquegua fuera del $1 \%$ entonces el impacto en los créditos de la región seria de $0,22 \%$; y si el crecimiento en el distrito fuera del $10 \%$, entonces el crecimiento en la región sería del 2,2\%.

- La ecuación lograda es: Log (Créditos total) = $0,514222839263+0,778253792255^{\star} \log$ (Créditos ILO) + 0,225467775099* Log (Créditos MOQUEGUA).

- El análisis histórico de los créditos, desde el año 2001 donde Ilo tuvo una participación del 87,3\% mientras que el distrito de Moquegua de 12,7\%. En el año 2019 el distrito de Ilo llega con un porcentaje de $68,8 \%$ y el distrito de Moquegua con $30,7 \%$ y el distrito de Omate con 0,25\% y se deja de contar.

- La media de los créditos en el periodo por distrito fue, en Ilo (73,9\%), Moquegua (25,7\%), Carumas $(0,1 \%)$, Omate $(0,2 \%)$ y por último el distrito de Torata con $0,1 \%$.

- Los créditos en Ilo si bien han tenido crecimientos anuales con desviaciones estándar notorios el promedio de crecimiento en el distrito de Ilo fue de $18,3 \%$ anual.

- ¿ ¿En cuántas veces los créditos de Ilo son mayores que los créditos colocados en Moquegua? En el 
año 2001 fue de 6,87 veces, así transcurrió a través de los años para llegar al año 2019 con una relación de 2,24 veces. Quiere decir que hoy los créditos de Ilo son 2,24 veces los colocados en Moquegua. La media de este indicador es de 3,3 veces. Quiere decir que el promedio de créditos colocados en Ilo, en los años 2001 al 2019 fue de 3,3 veces más que en el distrito de Moquegua.

- La diferencia de colocaciones de créditos entre los distritos de Moquegua e Ilo fue en el año 2001 de 84 millones. En el año 2019 fue de 580 millones de soles. Se concluye que la media de diferencia entre Ilo y Moquegua es de 235 millones de soles. (Ver tabla 4)

- La diferencia porcentual entre la colocación de créditos en Ilo y Moquegua llegó el año 2001 al $586 \%$. Quiere decir que la diferencia fue de 5,8 veces más. El año 2019 fue de 124,1\% la diferencia sustancial disminuyo.

- ¿ ¿Hay diferencias entre los créditos colocados en el distrito de Ilo y el distrito de Moquegua? Se entiende que una es mayor que la otra. En aplicación del ANVA se encuentra que el F calculado es 9,458 mayor que el F de las tablas. Se desprende, que sí hay diferencias entre los créditos colocados en el distrito de Ilo y Moquegua, porque la significancia es menor que $0,05 \mathrm{y}$ el dato de resultado es 0,004 .

Respecto a los depósitos por distritos en Moquegua, la correlación de Pearson (CP) entre depósitos en Ilo con los depósitos totales es, 99,5\%; lo mismo la CP de depósitos de Moquegua con respecto del total es de 98,9\%; los depósitos del distrito de Pacocha se relaciona con el total en 66\%; la CP de los depósitos del distrito de Torata con los ahorros totales de la región es de 87,5\%. Lo anterior indica que hay relación intensa entre los depósitos de 4 distritos respecto del total, desde el $66 \%$ al 99,5\%. Este último, corresponde al distrito de Ilo.

De la misma manera las CP de los ahorros en los distritos de Moquegua e Ilo es de 98,2\%. Lo cual nos da a entender que los ahorros de ambos distritos han seguido la misma dirección, quiere decir que mientras en un distrito crecía en el otro también, de tal manera que no pierde el paso del primer distrito.

De otro lado el crecimiento de los depósitos en la región se explica por el crecimiento de los ahorros en los distritos de Ilo, Moquegua y Pacocha. Para el caso se logró

Tabla 4

Total de créditos por distritos en la Región de Moquegua (en miles de soles)

\begin{tabular}{ccccccccc}
\hline Años & Ilo & Moquegua & Carumas & Omate & Torata & Pacocha & Puquina & Total \\
\hline 2001 & 98383 & 14324 & 0 & 0 & 0 & 2 & 0 & 112710 \\
2002 & 167696 & 18372 & 0 & 0 & 0 & 0 & 0 & 186068 \\
2003 & 140615 & 19487 & 0 & 0 & 0 & 1 & 0 & 160103 \\
2004 & 105585 & 24490 & 0 & 0 & 0 & 0 & 0 & 130075 \\
2005 & 117816 & 33854 & 0 & 0 & 0 & 0 & 0 & 151670 \\
2006 & 165560 & 42985 & 0 & 0 & 1 & 1 & 0 & 208546 \\
2007 & 326336 & 64372 & 0 & 0 & 87 & 1 & 0 & 390797 \\
2008 & 280927 & 91101 & 0 & 40 & 191 & 55 & 0 & 372314 \\
2009 & 312661 & 103552 & 0 & 315 & 280 & 58 & 0 & 416865 \\
2010 & 271606 & 118806 & 14 & 712 & 713 & 43 & 0 & 391893 \\
2011 & 338903 & 148084 & 457 & 988 & 1255 & 20 & 0 & 489707 \\
2012 & 364116 & 183899 & 2755 & 756 & 1491 & 6 & 0 & 553023 \\
2013 & 386896 & 215621 & 4080 & 2458 & 1433 & 0 & 0 & 610489 \\
2014 & 383676 & 239094 & 2977 & 3510 & 1955 & 0 & 101 & 631312 \\
2015 & 641635 & 284209 & 172 & 4140 & 740 & 0 & 0 & 930897 \\
2016 & 534965 & 323010 & 250 & 4880 & 880 & 0 & 409 & 864394 \\
2017 & 772467 & 355656 & 2232 & 5846 & 1085 & 0 & 0 & 1137285 \\
2018 & 1012326 & 406532 & 3035 & 6759 & 1037 & 0 & 621 & 1430310 \\
2019 & 1048182 & 467809 & 2625 & 3744 & 0 & 3 & 722 & 1523084 \\
\hline
\end{tabular}

Fuente: Elaboración propia en base a datos de la Superintendencia de Banca y Seguros (SBS) 
encontrar la siguiente regresión: Depósitos total $=4092,63$ $+0,9767^{\star}$ Depósitos ILO + 1,166*Depósitos Moquegua+ $1,088^{\star}$ Pacocha; su nivel de explicación está determinado por el $\mathrm{R}^{2}=0,9991$ que es bastante significativo. Respecto a los coeficientes de las variables se debe señalar que los betas de las variables son menores que 0,05 con lo cual deducimos que son coeficientes muy significativos.

Respecto al valor del F probabilístico (611145) de la tabla 3, nos indica que la ecuación interpreta de manera coherente el crecimiento del nivel de los depósitos totales de la región.

La elasticidad mide la variación proporcional de una variable respecto de la otra. Mide el impacto. En ese sentido la variación de los depósitos en el distrito de Ilo genera una variación en los depósitos totales. Si la variación es del $1 \%$ en los depósitos en Ilo, genera una variación de $0,75 \%$ en los ahorros totales de la región. Si hay una variación del 1\% en el distrito de Moquegua de los depósitos totales, entonces varia en $0,24 \%$ los depósitos en la región. De la misma manera si la variación fuese del $10 \%$ en los depósitos de Moquegua, entonces la variación en el depósito total regional es de 2,2\%. Esto demuestra que el mayor impacto causado es el incremento de los depósitos en Ilo.

De la misma manera si el incremento de los créditos en el distrito de Moquegua fuera del 1\%, entonces el impacto en los créditos de la región sería de $0,22 \%$, y si el crecimiento en el distrito fuera del $10 \%$, entonces el crecimiento de los depósitos en la región sería del 2,2\%.

Se nota que la regresión lograda es: LOG (Depósitos total $)=0,7318+0,757^{\star}$ LOG (depósitos ILO) $+0,2409^{\star} \mathrm{LOG}$ (depósitos MOQUEGUA). Al respecto el $\mathrm{R}^{2}=99,75 \%$, y los probabilísticos de los betas son menores de 0,05 que nos indica que son significativos.

En la serie historia que va del año 2001 al año 2019, se deja notar que en Ilo los depósitos tuvieron participación del 64,43\%; mientras que en el distrito de Moquegua eran de 23,3\%; en el distrito de Pacocha de $12,3 \%$ y en el distrito de Torata fue del $0 \%$. En el devenir de la data histórica se observa que al 2019, los porcentajes señalaban que el distrito de Ilo llega con un porcentaje de $57,1 \%$ y el distrito de Moquegua con 32,1\%, el distrito de Pacocha con el 7,1\% y el distrito de Torata con el 3,7\%.

La media de los depósitos (2001-2019) en el distrito de ILO fue de 59,2\%, en Moquegua de 28,4\%, en Pacocha de $7,4 \%$ y en Torata de $5 \%$. Los depósitos en Ilo, en los distritos, han tenido variabilidades extremas, de $-20 \%$ a crecimientos que superan el $137 \%$. Eso demuestra la volatilidad de esta variable pero que al final del periodo, se tiene que el crecimiento promedio anual en Ilo es de $11,2 \%$ y el crecimiento promedio anual en el distrito de Moquegua es de $16,5 \%$.
¿Cuán mayor son los depósitos en Ilo que los depósitos en el distrito de Moquegua? En el año 2001 fue de 2,8 veces, así trascurrió más o menos en los siguientes años de tal manera que en el año 2019 esta relación era de 1,8 veces. Quiere decir que hoy los depósitos de Ilo son 1,8 veces los depósitos en Moquegua. La media de este indicador es de 2,2 veces. Quiere decir que el promedio de ahorros de las familias y empresas en Ilo, en el periodo del 2001 al 2019 fue de 2,2 veces más que en el distrito de Moquegua.

La diferencia de depósitos en términos absolutos en los distritos de análisis Moquegua e Ilo son: en el año 2001 de 64,8 millones de soles; en el año 2019 de 268,4 millones de soles.

En aplicación del ANOVA se encuentra que el F calculado 12,261 es mayor que el F de las tablas. Se desprende porque la significancia es menor que 0,001 . El dato de resultado es 0,001 . Con lo anterior se concluye que sí hay diferencias entre los depósitos o ahorros, que también es el nivel de confianza lograda, en los distritos de Ilo y Moquegua.

\section{DISCUSIÓN}

La provincia de Ilo mejora su solidez financiera entre el 2001 al 2019. Si bien Galecio (2019) relaciona créditos y PBI, se demuestra que ante un incremento del 10\% de las colocaciones, el crecimiento del PBI sería de $0,69 \%$. Se demuestra que dado un incremento del $10 \%$ de los créditos en el distrito de Ilo, entonces los créditos en la región crecen en $6,6 \%$. Y la variación del $1 \%$ de los créditos en el distrito de Moquegua genera un impacto de $0,22 \%$ en los créditos totales de la región. En ese mismo sentido, Tapia (2018) señala que el incremento de los créditos sí influye en el PBI en el período 2009 al 2016, y que aplicando el modelo de crecimiento de Ramsey, demuestra que sí hay correlación entre estas variables, cuestión que fue aplicado para las 24 regiones. Se probó que si el incremento de los créditos fuese del $10 \%$ entonces el incremento de PBI seria del 6\% por el efecto elasticidad. Aguilar (2019), demostró que hay impacto de la expansión del crédito en el valor agregado y el desarrollo financiero en el periodo 2001-2008. Como mecanismo de efecto multiplicador los préstamos para vivienda son los de rápido efecto, así lo demostró Delgado (2019). Los créditos fortalecen la economía en crecimiento, lo dice Lizárraga (2014). Para ello se entrevistó a 80 funcionarios de alto nivel de las entidades financieras, y a la pregunta si la regulación del capital frenaba el crecimiento del crédito y al crecimiento económico del país, 72 de los 80 funcionarios respondieron que sí afectaba. Al cierre del año 2019 las entidades financieras se concentran en Ilo y Moquegua con 16 en cada una. Es de notar que en el año 2001 en la provincia de Ilo había 8 entidades financieras y en Moquegua solo tres. En el año 2009 en Ilo había 11 entidades financieras 
mientras que en Moquegua solo 7. Se puede decir que en el año 2020 se han equiparado en presencia de entidades financieras, mas no en volúmenes de créditos y depósitos. En la tesis de Quevedo (2018) se analiza que el crecimiento de la Cooperativa Juventud Ecuatoriana Progresista se explica por la normatividad legal. En Moquegua se demuestra que la Cooperativa Cuajone, la Cooperativa ILO y Santa Catalina representan el 25\% de los créditos totales. La tesis de Lage (2019) demuestra que los créditos sí pueden reactivar la economía de Ecuador; ya que encontró una correlación de 0,86 ; concluye que el sector financiero es importante para la economía del país. En Moquegua se observa que los créditos de Ilo tienen una correlación con los créditos de la región, en 99,5\% y lo explica en 99\%. De la misma manera Amézquita (2015) con vectores regresivos confirman que son los créditos de consumo lo que explica en mayor proporción el crecimiento del PBI de Colombia. En el caso que se analiza, dos distritos concentran el $70 \%$ de la capacidad de consumo de la región dejando atrás a otros 18 distritos. El 99,5\% de los créditos se concentran en dos distritos lo mismo que el 89,2\% de los depósitos. Los otros 18 distritos representan el 0,5\% en créditos y 10,8\% en depósitos.

\section{REFERENCIAS}

Amézquita, D. (2015). Ciclos económicos y crecimiento del crédito en Colombia (F. d. Universidad de los Andes, Ed.) Recuperada en https://repositorio.uniandes.edu. co/bitstream/handle/1992/13096/u714074.pdf?sequence $=1$ \&is Allowed $=\mathrm{y}$

Aguilar, G. (2019). Microcrédito y crecimiento regional en el Perú. Documento de trabajo 317. Recuperado de: http://repositorio.pucp.edu.pe/index/bitstream/ handle/123456789/46958/n_317.pdf?sequence $=1 \&$ isAllowed $=y$

Banco Central de Reserva del Perú. (12 de mayo de 2020). Informe Económico y Social Región Moquegua. Recuperado de: https://www.bcrp.gob.pe/docs/Proyeccion-Institucional/Encuentros-Regionales/2014/moquegua/ies-moquegua-2014.pdf

Delgado, A. (2019). La oferta del crédito hipotecario en el Perú en el periodo 2001-2009. Recuperado en http:// cybertesis.uni.edu.pe/bitstream/uni/3295/3/delgado_ cg.pdf.txt

Galecio, A. (2013). Impacto de la expansión de las colocaciones de las instituciones microfinacieras en el crecimiento regional del perú en el periodo 2001 - 2011(Tesis de pregrado). Universidad Privada Antenor Orrego. Recuperado de: http://repositorio.upao.edu.pe/bitstream/upaorep/151/1/GALECIO_ANDRE_IMPAC-
TO_EXPANSI\%c3\%93N_COLOCACIONES_INSTITUCIONES_CRECIMIENTO\%20REGIONAL.pdf

Instituto Nacional de Estadística e Informática. (12 de mayo de 2020). Moquegua: Resultados definitivos de la población economicamente activa. Recuperado en https://www.inei.gob.pe/media/MenuRecursivo/publicaciones_digitales/Est/Lib1624/18TOMO_01.pdf

Jurado Nacional de Elecciones. (18 de noviembre de 2002). Ley orgánica de Gobiernos Regionales Recuperado en https://portal.jne.gob.pe/portal_documentos/ files/informacionlegal/Constitucin\%20y\%20Leyes1/ LEY\%20ORGANICA\%20DE\%20GOBIERNOS\%20 REGIONALES.pdf

Lage, V. (2019). Reformas del Sistema Financiero y su Influencia sobre los Índices Económicos en el Ecuador Período 2010-2016 Recuperado en : http://repositorio. ucsg.edu.ec/bitstream/3317/10649/1/T-UCSG-POSMFEE-117.pdf

Lizárraga, R. (2019). El efecto del requerimiento de capital regulatorio de los bancos en el crecimiento del crédito que contribuye al desarrollo económico del país $R e$ cuperado en http://www.repositorioacademico.usmp. edu.pe/bitstream/usmp/1136/1/lizarraga_vrh.pdf

Miranda, F. Desigualdad, pobreza y migración en las provincias de Lima y el impacto del centralismo (2017). Investigaciones sociales, 20(37), pp. 131-147. Doi: https://doi.org/10.15381/is.v20i37.13432

Quevedo, L. (2018). Análisis comparativo de los factores que incidieron en el crecimiento de la cartera de la Cooperativa de Ahorro y Crédito Juventud Ecuatoriana Progresista y sus competidores (Tesis de maestría). Universidad Católica de Santiago de Guayaquil. Recuperado en http://repositorio.ucsg.edu.ec/bitstream/3317/11752/1/T-UCSG-POS-MFEE-136.pdf

Tapia, E. (2018). Microcrédito y crecimiento económico regional en Perú: 2009-2016 (Tesis para optar el título de Economista, Escuela Académico Profesional de Economía) Universidad Continental, Huancayo, Perú. Recuperado de: https://repositorio.continental.edu.pe/ bitstream/20.500.12394/4651/1/IV_FCE_313_TE_Tapia_Solis_2018.pdf 